\title{
An overview of infusing service-learning in medical education
}

\section{Trae Stewart, Zane Wubbena}

Department of Counseling, Leadership, Adult Education \& School Psychology, Texas State University, San Marcos, Texas, USA

Correspondence: Trae Stewart, Department of Counseling, Leadership, Adult Education \& School Psychology at Texas State University, 601 University Drive, ASBS 316, San Marcos, TX 78666, USA. Email: ps56@txstate.edu

\begin{abstract}
Objectives: To identify and review existing empirical research about service-learning and medical education and then to develop a framework for infusing service-learning in Doctor of Medicine or Doctor of Osteopathic Medicine curricula.
\end{abstract}

Methods: We selected literature on service-learning and medical education. Articles were screened with a protocol for inclusion or exclusion at two separate stages. At stage one, articles were screened according to their titles, abstracts, and keywords. The second stage involved a full-text review. Finally, a thematic analysis using focused and selective coding was conducted.
Results: Eighteen studies were analyzed spanning the years 1998 to 2012. The results from our analysis informed the development of a four-stage service-learning framework: 1) planning and preparation, 2) action, 3) reflection and demonstration, and 4) assessment and celebration.

Conclusions: The presented service-learning framework can be used to develop curricula for the infusion of servicelearning in medical school. Service-learning curricula in medical education have the potential to provide myriad benefits to faculty, students, community members, and university-community partnerships.

Keywords: Service-learning, medical education, experiential learning, community service, curriculum development

\section{Introduction}

Medical schools are challenged to better prepare future physicians to address the increasing and complicated healthcare needs of racially and culturally diverse societies. ${ }^{1-}$ ${ }^{3}$ As medical students transition into medical practitioners, they will encounter a growing number of patients whose health problems are the result of their environments. Impoverished communities, for example, encounter social, economic, and cultural factors that affect their health. These factors include, but are not limited to, infant mortality, asthma, obesity, mental health, drug/alcohol addictions, functional health and injuries, and children's readiness to learn in school. ${ }^{4}$ In these cases, medical students must be prepared to mitigate the effects of environmental multiplicities on the health of members of a society.

Correspondingly, medical school faculty must be prepared with instructional tools, strategies, and pedagogical know-how for helping future physicians understand and address the health problems encountered by members of communities in need. Unfortunately, medical school instructors' pedagogies have changed little over time; instructor-centered, direct instruction via lectures remains the dominant pedagogy in medical education. ${ }^{5}$ These instructor-centered lectures reduce students to mere recipients of knowledge, which often results in student boredom and a passivity toward learning new information. ${ }^{6}$

Pedagogies of engagement, like service-learning, empower students by providing them with an environment of authentic experiences that encourages critical thinking, problem-solving, and the application of knowledge. ${ }^{6,7}$ Further, experiential learning modalities contextualize knowledge beyond esoteric concepts and specialized skills learned in the classroom or laboratory. Service-learning's central components include: "Active participation, thoughtfully organized experiences, focus on community needs and school/community coordination, academic curriculum integration, structured time for reflection, opportunities for application of skills and knowledge, extended learning opportunities, and development of a sense of caring for others". 8

Service-learning's focus on community makes it applicable for preparing medical students to work in communities of need. The roots of service-learning can be traced to John Dewey. ${ }^{9}$ Dewey focused on preventing the tendency of the student to acquire stores of knowledge useless in new 
situations. He noted that education of social value could not take place in the abstract (e.g., classroom, clinic). Rather, Dewey suggested that "educative [experiences] must lead out into an expanding world".,10 The community is an educative laboratory for the application of knowledge. ${ }^{10}$ Students can discover relationships among ideas for themselves, rather than being passive recipients of prescriptive information.

Dewey's philosophy of experience appears suited as a key pedagogical element that underpins instructional change in medical education. Medical students must understand the effects of the environment on patient wellbeing. And, medical schools can provide opportunities for students to expand their knowledge into the community through service-learning. The purpose of this study was to identify existing empirical literature on service-learning and medical education. Then, we aimed to develop an overview framework for infusing service-learning in Doctor of Medicine or Doctor of Osteopathic Medicine curricula.

\section{Methods}

\section{Search strategy}

A literature search was conducted for this study, and thus, no formal ethical approval was required from our university's Institutional Review Board (IRB). First, a literature search of databases available internally through our university library were queried for the English language keywords and Boolean combinations (use of " "denotes the search for exact terms): "medical education" AND "service learning"; "medical education" AND "service-learning"; "medical school" AND "service-learning"; "medical school" AND "service learning"; "medical students" AND "service learning"; "medical students" AND "service-learning." These databases were EBSCOHost, ProQuest, ERIC, JSTOR, Education Source, and dissertation/thesis abstract search engines. Then, external databases were searched: National Service-Learning Clearinghouse, Google Scholar, MEDLINE, and PubMed. Full-text PDFs of articles were saved.

\section{Inclusion and exclusion process}

A screening protocol for the review of the articles was developed. Articles were subjected to a two-stage inclusion and exclusion screening. The first stage of the screening process required the review of each article's title, abstract, and keywords. The inclusion criteria included empirical studies pertaining to medical students working toward a Doctor of Medicine (M.D.) or a Doctor of Osteopathic Medicine (D.O.) degree who engaged in service-learning. Articles reporting community service alone and/or servicelearning in other health services (e.g., nursing, physician assistants, dentistry, pharmacy, occupational and physical therapy) were excluded because these topics exceeded the inclusion criteria parameters. Then we applied the inclusion and exclusion criteria to the full text each article at stage two of the screening process.

\section{Data abstraction and analysis}

A thematic analysis was employed in order to inductively translate textual data into recurring themes for meaningful analysis. Our coding occurred at two levels. First, we used focused coding to develop categories and connections between central concepts. ${ }^{11}$ Each time a theme was identified, the text of each article was re-read. The themes included: course type, service required, participants, objectives, methods/interventions, student assessments, and results/outcomes. Then, we used selective coding for the development of subcategories related to each of the major themes identified. For example, the theme, course type, included: required, elective, and selected courses. We then synthesized the themes and subthemes together into an overview framework for infusing service-learning into medical education.

\section{Results}

Sixty-three studies were identified using the search strategy of Boolean keyword combinations. After stage one of the inclusion and exclusion screening (i.e., review of each study's title, abstract, and keywords), a total of 16 studies was excluded. Stage two included the full-text screening of the 47 remaining articles. After stage two, a total of 29 additional studies was excluded. The selective-sample of articles qualifying for final analysis included 18 studies spanning the years from 1998 to 2012. The inter-rater agreement for each independently reviewed full-text article was $100 \%$. Table 1 presents summarized data from the studies that qualified for the final review. ${ }^{12-29}$

Based on the review of the articles and the thematic analysis, an overview of four stages was developed for infusing service-learning in medical education. This overview was composed of the following stages: 1) planning and preparation (four subcategories); 2) action (two subcategories); 3) reflection and demonstration (one subcategory); and, 4) assessment and celebration (four subcategories).

\section{Planning and preparation}

\section{Differentiate between service-learning and community service}

Service-learning encompasses a reciprocal interaction between service to the community and learning tied to specific course objectives. Conversely, service is often used in the form of episodic community-service or volunteering activities (e.g., logging community service hours to graduate; compensatory service assigned by the courts; or benefiting only students or community). One-sided activities, although beneficial in many respects, may actually detract from the potential educational benefits of service-learning when integrated into the medical education curriculum; this is especially the case when service-learning is an "add-on" to the curriculum. 
Decide when and where to infuse service-learning into curriculum

The potential years and courses in which to infuse service-learning into the medical education curriculum depends on the course goals and objectives to be achieved. Course goals and objectives may include:

- Society includes a focus on the social interests of larger society and local communities;

- Eternal and divine sources perpetuate society by linking values, ethics, and morality;

- Science includes what can be observed and quantified;

- Knowledge of a discipline that has a particular method used to extend its boundaries; and,

- Learners include what we know of student's cognition, and psychosocial development.

The selection of the goal coupled with whether the course is required and the knowledge level of participating students, informs the year of study in which students participate in service-learning. Introducing service-learning during the M1 year is important: students who had the opportunity to begin their medical education with a service-learning opportunity were more likely to carry a community-based perspective throughout their medical training, while simultaneously building a renewable pool of students/servicelearners in their medical schools. ${ }^{15}$

\section{Build university-community partnerships}

Effective service-learning relies on strong campuscommunity partnerships. These partnerships can manifest in many different variations, from institution-community, to individual course-community, and even to student organization-community. Each level of integration depends on the formality, goal, type of learning, and communitypartner relationship. During the planning and preparation phase, agreements are made between the service-learning designer/instructor, service-learners/students, and the community members/organization. Students and community partners should be involved at the start to contribute to the planning process, to know what is expected, and to understand the desired outcomes from the service.

The key to incorporating service-learning programs that have the ability to evolve over time is to establish relationships of trust with community-based organizations. ${ }^{30}$ Personal relationships and experience working together are often necessary for forming service-learning partnerships. Faculty usually work with non-profits to which they, or their colleagues, are already connected, as the trust and genuineness are usually developed already. Another approach is to build on existing campus-community partnerships for which a memorandum of understanding (MoU) exists. Many medical education programs have memoranda of understanding with community engagement or volunteer offices charged with maintaining partnerships. The community-institution partnership can too be enhanced through the co-evaluation of the service-learning program to ensure the relationship shares a common interest, complementary skills and resources, and a commitment to the instruction and assessment of medical students.

Overtime, the effect of service-learning programs will contribute to building relationships of trust with community-based organizations. Relationships of trust are built though the willingness of the institution, faculty, students, and community-based organizations to develop shared values. ${ }^{31}$ In order to ensure that the values of the community-based organizations are aligned with the values of the participants of the service-learning program, it is important to include community partners as co-evaluators who can provide feedback on student performance. ${ }^{32}$ This feedback allows both students and faculty to consider the perspective of the community when determining ways to improve the service-learning program.

\section{Establish structure, funding, and recognition for faculty} Service-learning institutionalization requires structural (offices and policies), procedural (activities and cultural mindset), and integration. A lack of time is one pervasive explanation as to why faculty have decided not to infuse service-learning, or to stop requiring service-learning. ${ }^{14}$ Unlike more traditional pedagogies in which the professor prepares information to be presented to a large group of students at one time (i.e., lecture-based instruction), service-learning requires faculty to find partners, develop reflection activities and assessments to match the course objectives, juggle each student's individual experiences, liaise with professionals at the university to create MoUs to manage risk and liability, identify readings that incorporate community health and civic elements, all while working with other faculty and dozens of students. It is understandable that faculty who must complete these additional tasks may opt-out unless provided with the necessary time and support. Dedicated time is especially crucial for the firsttime implementation of service-learning. However, longitudinal service-learning projects may both decrease the overall time commitment and increase program sustainability. 33,34

Another major limitation to service-learning that affects implementation and sustainability is funding. Faculty must "sell" the concept to campus administrators by demonstrating how service is contributing to the mission, curriculum objectives, and research of the institution. A first step is to establish an engagement taskforce by including individuals familiar with community engagement and who can draft an agenda as a guide for over five years. One approach is for faculty to design service-learning programs that aim to generate funding and become self-sustainable by holding fundraising events aimed at addressing community needs. ${ }^{35}$ Another approach is for faculty to designing servicelearning activities where students go into the community and meet with families at their home. Home-based service 
learning provides students with an opportunity to spend individualized time with patients while also providing students with first-hand experience of common environmental/living health concerns. ${ }^{33,34}$

Table 1. Study characteristics of service-learning in medical school

\begin{tabular}{|c|c|c|c|c|c|c|c|}
\hline Study & Course type & $\begin{array}{l}\text { Service } \\
\text { required }\end{array}$ & $\begin{array}{l}\text { Partici- } \\
\text { pants }\end{array}$ & $\begin{array}{l}\text { Aim or } \\
\text { objective }\end{array}$ & Methods or intervention & $\begin{array}{l}\text { Student } \\
\text { assessment }\end{array}$ & Results or outcomes \\
\hline $\begin{array}{l}\text { Averill } e t \\
\text { al., } 2007^{12}\end{array}$ & Elective & 32 hours & $\begin{array}{l}\text { M1 } \\
(\mathrm{N}=7)\end{array}$ & $\begin{array}{l}\text { Health } \\
\text { behavior } \\
\text { education }\end{array}$ & $\begin{array}{l}\text { Needs-assessment, } \\
\text { health education } \\
\text { intervention, interviews } \\
\text { and questionnaires }\end{array}$ & Reflective essay & $\begin{array}{l}\text { - Developed collaboration skills } \\
\text { - Improved communication skills } \\
\text { - Increased sex education knowledge } \\
\text { - Identified community needs } \\
\text { - Exceeded required service hrs. }\end{array}$ \\
\hline $\begin{array}{l}\text { Buckner et } \\
\text { al., } 2010^{13}\end{array}$ & Required & 1 year & M1 & $\begin{array}{l}\text { Health } \\
\text { behavior } \\
\text { education }\end{array}$ & $\begin{array}{l}\text { Needs-assessment, } \\
\text { health education } \\
\text { intervention, presenta- } \\
\text { tions of outcomes, and } \\
\text { policy recommenda- } \\
\text { tions for community }\end{array}$ & $\begin{array}{l}\text { Reflective essay, } \\
\text { reflective } \\
\text { discussion, } \\
\text { multiple-choice } \\
\text { test, attendance }\end{array}$ & $\begin{array}{l}\text { - Developed collaboration skills } \\
\text { - Implemented health behavior interventions } \\
\text { - Learned clinical skills related to dementia } \\
\text { and Alzheimer's } \\
\text { - Addressed health-needs of underserved } \\
\text { community } \\
\text { - Developed teaching skills }\end{array}$ \\
\hline
\end{tabular}

\begin{tabular}{|c|c|c|c|c|c|c|c|}
\hline $\begin{array}{l}\text { Burrows et } \\
\text { al., } 1999^{14}\end{array}$ & Required & 20 hours & $\begin{array}{l}\text { M1 and } \\
\text { M2 }\end{array}$ & $\begin{array}{l}\text { Identify and } \\
\text { address } \\
\text { needs of } \\
\text { community }\end{array}$ & $\begin{array}{l}\text { Select project at school } \\
\text { fare: clinical services, } \\
\text { education, fund-raising, } \\
\text { behavior interventions, } \\
\text { obtain feedback for } \\
\text { qualitative analysis }\end{array}$ & $\begin{array}{l}\text { Hour-tracking of } \\
\text { service, } \\
\text { questionnaire } \\
\text { (open-ended) }\end{array}$ & $\begin{array}{l}\text { - Increased communication skills } \\
\text { - Developed compassion, respect, and comfort } \\
\text { working with underserved population } \\
\text { - Learned clinical skills: taking blood pressure } \\
\text { and testing for tuberculosis } \\
\text { - Served as role models for youth } \\
\text { - Exceeded volunteer hours }\end{array}$ \\
\hline $\begin{array}{l}\text { Elam et al., } \\
2003^{15}\end{array}$ & Elective & $\begin{array}{l}18 \text { hours } \\
\text { (each year) }\end{array}$ & $\begin{array}{l}\text { M1 and } \\
\text { M2 }\end{array}$ & $\begin{array}{l}\text { Health } \\
\text { behavior } \\
\text { education }\end{array}$ & $\begin{array}{l}\text { Address community } \\
\text { needs by using } \\
\text { community assets }\end{array}$ & $\begin{array}{l}\text { Reflective essay, } \\
\text { questionnaire }\end{array}$ & $\begin{array}{l}\text { - Identified community assets } \\
\text { - Used community assets to address commu- } \\
\text { nity health needs }\end{array}$ \\
\hline $\begin{array}{l}\text { Jefferson } \\
\text { et al., } \\
2012^{16}\end{array}$ & Elective & 1 year & $\begin{array}{l}M 1 \\
(N=45)\end{array}$ & $\begin{array}{l}\text { Alzheimer's } \\
\text { education }\end{array}$ & $\begin{array}{l}\text { Paired students with } \\
\text { early-stage Alzheimer's } \\
\text { patients, conducted } \\
\text { pretest-posttest of } \\
\text { knowledge related to } \\
\text { Alzheimer's disease } \\
\text { and dementia }\end{array}$ & $\begin{array}{l}\text { Reflective essay, } \\
\text { specialty test } \\
\text { (Alzheimer's } \\
\text { disease and } \\
\text { dementia } \\
\text { knowledge) }\end{array}$ & $\begin{array}{l}\text { - Increased communication skills } \\
\text { - Developed clinical skills related to dementia } \\
\text { and Alzheimer's } \\
\text { - Increased future likelihood of providing } \\
\text { geriatric care } \\
\text { - Learned about personal factors (e.g., family) } \\
\text { affecting patients with Alzheimer's disease }\end{array}$ \\
\hline $\begin{array}{l}\text { Leung et } \\
\text { al., } 2007^{17}\end{array}$ & Required & 6 weeks & $\begin{array}{l}\text { M1 } \\
(N=249)\end{array}$ & $\begin{array}{l}\text { Attitudes } \\
\text { toward } \\
\text { healthcare } \\
\text { and } \\
\text { knowledge } \\
\text { of health } \\
\text { issues }\end{array}$ & $\begin{array}{l}\text { Participation in two- } \\
\text { week training, live in } \\
\text { underserved community } \\
\text { while providing home- } \\
\text { based and clinic-based } \\
\text { healthcare }\end{array}$ & $\begin{array}{l}\text { Reflective essay, } \\
\text { reflective } \\
\text { discussion, } \\
\text { questionnaire }\end{array}$ & $\begin{array}{l}\text { - Increased leadership skills } \\
\text { - Increased communication skills } \\
\text { - Developed students' sense of social justice } \\
\text { - No increase in critical thinking } \\
\text { - Less confidence providing healthcare } \\
\text { independently }\end{array}$ \\
\hline $\begin{array}{l}\text { Long et al., } \\
2011^{18}\end{array}$ & Elective & $\begin{array}{l}8 \text { weeks } \\
\text { (data over } 5 \\
\text { years) }\end{array}$ & $\begin{array}{l}\mathrm{M} 1 \\
(\mathrm{~N}=62)\end{array}$ & $\begin{array}{l}\text { Leadership } \\
\text { skills and } \\
\text { community } \\
\text { advocacy }\end{array}$ & $\begin{array}{l}\text { Students paired with } \\
\text { faculty mentors and } \\
\text { community-based } \\
\text { organizations. They } \\
\text { completed an advocacy } \\
\text { course, internship, and } \\
\text { scholarly activity. }\end{array}$ & Questionnaire & $\begin{array}{l}\text { - Increased leadership skills } \\
\text { - Increased teaching skills } \\
\text { - Developed an understanding of health } \\
\text { disparities that could be addressed by health } \\
\text { education interventions, community partner- } \\
\text { ships, and changes to policy/legislative } \\
\text { mandates }\end{array}$ \\
\hline $\begin{array}{l}\text { McConnell } \\
\text { et al., } \\
2010^{19}\end{array}$ & Required & $\begin{array}{l}1 \text { year } \\
\text { (data over } 3 \\
\text { years) }\end{array}$ & $\begin{array}{l}M 1 \\
(N=301)\end{array}$ & $\begin{array}{l}\text { Pediatric } \\
\text { knowledge }\end{array}$ & $\begin{array}{l}\text { Lectures on health } \\
\text { topics, provide physical } \\
\text { exams to students from } \\
\text { inner-city schools }\end{array}$ & $\begin{array}{l}\text { Pediatric exams, } \\
\text { questionnaire }\end{array}$ & $\begin{array}{l}\text { - Learned to identify and address the health- } \\
\text { needs of an underserved community } \\
\text { - Increased teaching and presentation skills }\end{array}$ \\
\hline $\begin{array}{l}\text { McNeal et } \\
\text { al., } 2012^{20}\end{array}$ & Selective & $\begin{array}{l}2 \text { to } 3 \\
\text { months }\end{array}$ & $\begin{array}{l}\mathrm{M} 1, \mathrm{M} 2 \\
\mathrm{M} 3 \text {, and } \\
\mathrm{M} 4\end{array}$ & $\begin{array}{l}\text { Health } \\
\text { behavior } \\
\text { education }\end{array}$ & $\begin{array}{l}\text { Mini-grants awarded to } \\
\text { student groups, groups } \\
\text { conducted needs- } \\
\text { assessments, held } \\
\text { health a fare, and } \\
\text { developed summer } \\
\text { projects to address } \\
\text { community needs }\end{array}$ & $\begin{array}{l}\text { Poster presenta- } \\
\text { tions, question- } \\
\text { naire }\end{array}$ & $\begin{array}{l}\text { - Developed leadership skills } \\
\text { - Increased understanding of the social } \\
\text { determinants of health } \\
\text { - Developed understanding of health dispari- } \\
\text { ties that could be addressed by health } \\
\text { education interventions, community partner- } \\
\text { ships, and changes to policy/legislative } \\
\text { mandates }\end{array}$ \\
\hline $\begin{array}{l}\text { Meili et al., } \\
2011^{21}\end{array}$ & Selective & 2 years & $\begin{array}{l}\text { M1 and } \\
\text { M2 } \\
(\mathrm{N}=14)\end{array}$ & $\begin{array}{l}\text { Develop } \\
\text { social } \\
\text { accountabil- } \\
\text { ity }\end{array}$ & $\begin{array}{l}\text { Service-learning } \\
\text { program rotates through } \\
\text { urban, rural, and } \\
\text { international locations } \\
\text { (students learn foreign } \\
\text { language), qualitative } \\
\text { data analysis conducted } \\
\text { on open-ended } \\
\text { questionnaires }\end{array}$ & Reflective essay & $\begin{array}{l}\text { - Increased importance of patient-physician } \\
\text { relationships } \\
\text { - Developed multicultural understanding of } \\
\text { community } \\
\text { - Increased understanding of the social } \\
\text { determinants of health } \\
\text { - Increased sense of social justice } \\
\text { - Recognized need to affect unhealthy habits } \\
\text { of communities } \\
\text { - Mixed results on students selection of } \\
\text { medical specialty }\end{array}$ \\
\hline $\begin{array}{l}\text { Packer et } \\
\text { al., } 2010^{22}\end{array}$ & Elective & 4 days & $\begin{array}{l}M 3 \\
(N=53)\end{array}$ & $\begin{array}{l}\text { Community } \\
\text { develop- } \\
\text { ment }\end{array}$ & $\begin{array}{l}\text { Visit homeless clinics } \\
\text { and shelters, engage in } \\
\text { street outreach, results } \\
\text { analyzed quantitatively } \\
\text { and qualitatively }\end{array}$ & $\begin{array}{l}\text { Reflective essay, } \\
\text { reflective } \\
\text { discussion, } \\
\text { questionnaire }\end{array}$ & $\begin{array}{l}\text { - Greater understanding of the legal issues } \\
\text { and bureaucratic barriers facing healthcare } \\
\text { - Increased sense of social justice } \\
\text { - Exceeded volunteer hours } \\
\text { - Mixed results on students selection of } \\
\text { medical specialty }\end{array}$ \\
\hline
\end{tabular}




\begin{tabular}{|c|c|c|c|c|c|c|c|}
\hline $\begin{array}{l}\text { Sakai et } \\
\text { al., } \\
2002^{23}\end{array}$ & $\begin{array}{l}\text { Elective, } \\
\text { Selective }\end{array}$ & 1 year & $\begin{array}{l}M 1 \\
(N=5)\end{array}$ & $\begin{array}{l}\text { Health } \\
\text { behavior } \\
\text { education }\end{array}$ & $\begin{array}{l}\text { Students created } \\
\text { curriculum, lessons on } \\
\text { health topics at a high } \\
\text { school, pretest-posttest } \\
\text { design }\end{array}$ & $\begin{array}{l}\text { Specialty test } \\
\text { (sex education) }\end{array}$ & $\begin{array}{l}\text { - Increased sex education knowledge } \\
\text { - Increased teaching and presentation skills } \\
\text { - Increased sense of social justice }\end{array}$ \\
\hline $\begin{array}{l}\text { Steiner } e t \\
\text { al., } 2000^{24}\end{array}$ & Elective & 1 week & $\begin{array}{l}\mathrm{M} 2 \\
(\mathrm{~N}=71)\end{array}$ & $\begin{array}{l}\text { Natural } \\
\text { disaster } \\
\text { mobilization } \\
\text { and relief }\end{array}$ & $\begin{array}{l}\text { Community needs- } \\
\text { assessments developed } \\
\text { by not-for-profit } \\
\text { organizations used by } \\
\text { student groups to } \\
\text { provide various relief } \\
\text { efforts after a natural } \\
\text { disaster }\end{array}$ & $\begin{array}{l}\text { Reflective essay, } \\
\text { reflective } \\
\text { discussion, } \\
\text { questionnaire }\end{array}$ & $\begin{array}{l}\text { - Improved teamwork } \\
\text { - Increased leadership skills } \\
\text { - Improved communication } \\
\text { - Identify other health-needs of underserved } \\
\text { community } \\
\text { - Understanding of challenges facing commu- } \\
\text { nity organizations } \\
\text { - Understanding of the social determinants of } \\
\text { health problems } \\
\text { - Understanding of communities coping with } \\
\text { natural disasters }\end{array}$ \\
\hline $\begin{array}{l}\text { Stearns et } \\
\text { al., } 2000^{25}\end{array}$ & Selective & 1 year & $\begin{array}{l}\text { M4 } \\
(N=112)\end{array}$ & $\begin{array}{l}\text { Health } \\
\text { factors of a } \\
\text { rural } \\
\text { community }\end{array}$ & $\begin{array}{l}\text { Longitudinal design, } \\
\text { students wanting to } \\
\text { work in family } \\
\text { healthcare located in a } \\
\text { rural area }\end{array}$ & $\begin{array}{l}\text { Poster presenta- } \\
\text { tion }\end{array}$ & $\begin{array}{l}\text { - Identified health needs of underserved } \\
\text { community } \\
\text { - Increased comfort with using health-related } \\
\text { technology } \\
\text { - Increased understanding of limitations that } \\
\text { affect rural community health }\end{array}$ \\
\hline $\begin{array}{l}\text { Switzer, } \\
1999^{26}\end{array}$ & Selective & 1 year & $\begin{array}{l}M 1 \\
(N=26)\end{array}$ & $\begin{array}{l}\text { Psychoso- } \\
\text { cial behavior } \\
\text { of females } \\
\text { who are } \\
\text { pregnant }\end{array}$ & $\begin{array}{l}\text { Pretest-posttest, } \\
\text { Paired } 26 \text { first year med } \\
\text { students with pregnant } \\
\text { females who were low- } \\
\text { income adolescents }\end{array}$ & $\begin{array}{l}\text { Specialty test } \\
\text { (prenatal care), } \\
\text { questionnaire }\end{array}$ & $\begin{array}{l}\text { - Learned clinical skills: Testing blood } \\
\text { pressure and for tuberculosis }\end{array}$ \\
\hline $\begin{array}{l}\text { O'Tool } e t \\
\text { al., } 2005^{27}\end{array}$ & $\begin{array}{l}\text { Elective, } \\
\text { Selective }\end{array}$ & $\begin{array}{l}\text { M1 } \\
\text { (pre-clinical } \\
8 \text { weeks) } \\
\text { M3 and M4 } \\
\text { (clinical } 1 \\
\text { month) }\end{array}$ & $\begin{array}{l}\mathrm{M} 1, \mathrm{M} 3 \\
\text { and } \mathrm{M} 4 \\
(\mathrm{~N}=95)\end{array}$ & $\begin{array}{l}\text { Community } \\
\text { develop- } \\
\text { ment }\end{array}$ & $\begin{array}{l}\text { Students assigned to a } \\
\text { community organization } \\
\text { and work with a } \\
\text { community mentor. } \\
\text { Students developed } \\
\text { health projects (e.g., } \\
\text { health needs survey, } \\
\text { health brochures) }\end{array}$ & $\begin{array}{l}\text { Reflective essay, } \\
\text { reflective } \\
\text { discussion, } \\
\text { questionnaire } \\
\text { (open-ended) }\end{array}$ & $\begin{array}{l}\text { - Learned to identify and address the health- } \\
\text { needs of an underserved community }\end{array}$ \\
\hline $\begin{array}{l}\text { Waddell et } \\
\text { al., } 2000^{28}\end{array}$ & Required & 1 year & $\begin{array}{l}\mathrm{M} 1 \\
(\mathrm{~N}=85)\end{array}$ & $\begin{array}{l}\text { Health } \\
\text { behavior } \\
\text { education }\end{array}$ & $\begin{array}{l}\text { Identification of } \\
\text { resources and devel- } \\
\text { opment of plans to } \\
\text { prevent illness and } \\
\text { stabilize of chronic } \\
\text { illness. }\end{array}$ & $\begin{array}{l}\text { Specialty test } \\
\text { (Genogram tool) }\end{array}$ & $\begin{array}{l}\text { - Improved teamwork } \\
\text { - Greater understanding of home-based } \\
\text { factors affecting health }\end{array}$ \\
\hline $\begin{array}{l}\text { Wee et al., } \\
2011^{29}\end{array}$ & Elective & $\begin{array}{l}1 \text { year } \\
\text { (optional } \\
\text { project } \\
\text { swap mid- } \\
\text { year) }\end{array}$ & $\begin{array}{l}M 1 \\
(N=824)\end{array}$ & $\begin{array}{l}\text { Service- } \\
\text { learning: } \\
\text { home-based } \\
\text { vs. clinic- } \\
\text { based }\end{array}$ & $\begin{array}{l}\text { Cross-comparison } \\
\text { design between clinic- } \\
\text { based and home-based } \\
\text { service-learning using } \\
\text { self-administered } \\
\text { questionnaires }\end{array}$ & $\begin{array}{l}\text { Self- } \\
\text { administered } \\
\text { questionnaire }\end{array}$ & $\begin{array}{l}\text { - Improved teamwork } \\
\text { - Improved communication } \\
\text { - Developed knowledge of disease manage- } \\
\text { ment } \\
\text { - Greater understanding of the home-based } \\
\text { factors affecting health } \\
\text { - Increased understanding of the social } \\
\text { determinants of health }\end{array}$ \\
\hline
\end{tabular}

To recognize engaged faculty, a service-learning scholar/fellows programs can be established. These competitive programs name a couple of faculty each year as "engaged scholars" who are charged with assisting their colleagues to design service-learning modules and an associated research agenda. Often, the scholars receive acknowledgement, a cash stipend, travel monies for professional development, and/or course release time for service, while the faculty who are implementing service-learning may also receive a cash stipend or course release for one semester. Another recognition event is to consider making "engagement" a theme for an academic year. A taskforce may recommend readings to faculty, invite guest speakers, hold joint campuscommunity activities, and host visiting fellows for trainings/workshops. Students may present their own research conducted during this time at an end-of-year poster fair.

\section{Action}

Match service activity to learning goal(s)

Action is the service activity itself. Service-learning activities in medical education can be categorized into three broad types: 1) educational/training (e.g., teaching CPR to young parents, health behavior intervention programs in schools), 2) clinic-/community-based (e.g., health fair for elderly, Alzheimer patient care), 3) advocacy, policy, and outreach (e.g., Autism awareness, fundraising). ${ }^{36}$ Indirect (e.g., organizing first aid kits) and research projects are also service options. Regardless of type, the service must be meaningful, have academic integrity (i.e., not just thrown into a course), put the student in a position of responsibility and ownership, allow for project supervision and mentoring, place community organizations as co-instructors in student learning, and be appropriate for the needs being addressed as well as the developmental levels of the participants. Attention should be taken to ensure that the action, learning goals and objectives, and community needs are aligned.

\section{Program design and student participation}

The design of the service-learning course can be elective, requisite, or selection-based. From this review, servicelearning elective courses are the most prominent, but often 
restrict participation to those students who self-selected into the course and may be, arguably, already service-oriented. Selection-based service-learning courses often require an application and acceptance in order to participate. Both elective and selection courses may provide flexibility for when student participation is limited. In order to reach all students, programs may choose to require participation in service-learning by infusing the pedagogy into the required curriculum; this allows the concept of altruism to be introduced more broadly across the diverse student population. Required service-learning is likely to be sustained over time through an ongoing volunteer of new students. In addition, sustained service-learning programs reduce the costs associated with program start-up (e.g., materials, resources, faculty training).

Level of student performance also affects servicelearning involvement. Brush, Markert, and Lazarus ${ }^{37}$ found that students in the lower and higher quartiles of class rank were less likely to participate in service-learning, while students in the middle quartiles (i.e., 66\%) were more likely to engage in service-learning. Perhaps integrating a mandatory service-learning course may ameliorate performance issues of struggling students by providing them with the opportunity to learn by doing, while providing students ranked in the higher quartiles of their class with opportunities to develop communication skills by increasing personal interactions with community members. This opportunity is mutually beneficial-that is, community members can benefit from the service-learner's expertise while the latter gains an increased understanding of the contextual problem that faced the health and wellness of an underserved community. ${ }^{37}$

\section{Reflection and demonstration}

\section{Best practices for organizing reflection}

Reflection is the conduit between volunteer service, academic coursework, and civic intentions-the glue holding service and learning together. ${ }^{38}$ Reflections should be continuous (i.e., before, during, after), connected to academic and real-life needs (learning objectives for synthesizing action and thought), challenging to prompt critical thinking, contextualized within the course and service setting, and involve communication with peers, instructors, and representatives from the organization. Reflection examples include: discussion, presentations/performance, journals/writings, role-play, artwork/portfolios, research posters, and meditation.

Best practices for reflection in service-learning include: setting goals, knowing the audience, making time, choosing a method, sharing expectations, identifying resources, reviewing skills, creating transparent evaluations, demonstrating the importance of different types of reflection, and embracing/capitalizing on teachable moments. Students engaged in service-learning need to also process more than academic topics during their service experiences; additional reflection on civic engagement, stereotypes, feelings of privilege, and emotional distress is warranted. The following barriers to reflection should be avoided: reflection as an extra activity; relegating reflection toward the end of the semester; framing reflection as an individual activity or log of events; and, failing to encourage students to take a selfsearching and critical stance.

\section{Assessment and celebration}

\section{Qualitative and quantitative assessment}

Assessment is necessary to determine student outcomes, document community impacts, and evaluate program structure and process, including the relationship between the community and medical institution. Quantitative measures, such as clinical-knowledge tests, attendance, and multiple-choice exams, placed outcomes in a predetermined context. This context represented the skills, knowledge, or experiences that students were expected to have learned. A starting point for research instruments is the Compendium of Assessment and Research Tools (CART) and Project STAR. Lastly, a compendium of scales commonly used in service-learning is available in The Measure of Service Learning. ${ }^{39}$ These resources can help faculty locate assessment tools that are aligned with their course/program objectives.

On the other hand, qualitative measures can illuminate unexpected outcomes that may lead to greater program flexibility, if not innovation. Qualitative measures include written reflection, reflective discussion, journaling, written essays, interviews, portfolios, and open-ended questions. Poster presentations are an effective culminating activity when used to share the service-learning experience with medical school faculty, students, and community members. $^{40}$

\section{Student outcomes}

Student outcomes help to determine if the objectives of the service-learning activity were met. It is important to consider and report the descriptive variables (i.e., course objectives, duration, service activity, funding, community partners, participants, types of reflection, and type of assessment) in order to frame and place the results in context. Three themes of student outcomes were identified from the literature: 1) academic learning and professional development, 2) personal development, and 3) enhanced civism and social responsibility.

First, the students involved in service-learning demonstrated increased academic learning and professional responsibility. This academic learning included both nonclinical and clinical skills, which often depended on the course and the student's year in medical school when participating in service-learning. Increased self-confidence and efficacy and the development of problem analysis skills were also beneficial outcomes of students' participation in 
service-learning. Further, students appeared to develop a better understanding of their roles as physicians and of professional work environments, while also developing a better understanding of public health and the impact of health legislation and policies.

Next, outcomes also resulted in changes to students' intrapersonal and interpersonal skills and leadership ability. Students immersed in the community developed their communication skills with patients, community members, other students, and school faculty. They were able to understand diversity while showing increased levels of compassion and respect for others from different backgrounds. Students also demonstrated increased teamwork, collaboration, and leadership skills. And, many became more likely to step in and take on the responsibilities associated with leadership.

Lastly, students reported increased social responsibility and civism to support the underserved community. They demonstrated an increased sense of social justice and found many of the health related problems embedded in a perpetual cycle that was difficult to break. Students often found themselves eagerly engaging in more hours of service than were required. Finally, service-learners were able to support communities by helping members of these communities understand both their assets and their needs.

\section{Encourage the scholarship of teaching and learning}

Medical school faculty and medical school students should actively engage in scholarship aimed at reporting effective and ineffective teaching and learning practices in servicelearning. The Scholarship of Teaching \& Learning (SoTL) involves the study of teaching and/or learning and the public sharing and review of such work through presentations, performance, or publications. ${ }^{41}$ Instructors can cowrite/publish with community partners and students. The publication of original scholarship in scholarly peerreviewed journals is important to clearly articulate the methods, results, and outcomes for the evaluation and comparison of service-learning programs. Further inquiry on the topics/designs for research include: epistemological models applied to service-learning, impact of student knowledge, longitudinal/time-series, control-group designs, pretest-posttest designs, community impact studies with community groups/partners, cross-disciplinary studies connecting quantitative and qualitative outcomes, and internationally comparative studies. The improvement of service-learning in medical school is dependent on sharing examples and outcomes vis-à-vis both teaching and learning.

\section{Celebrate, recognize, and share the learning}

Celebration is an opportunity to acknowledge the work, learning, and appreciation of the service-learning activity. As noted in SoTL above, there is a public aspect to servicelearning, which can be emphasized through special media coverage and newspaper articles written by participants, or by having everyone sign a symbolic agreement to continue the work. Students with research interests can present posters at fairs put on by the medical school or at professional academic conferences. For positive reinforcement, students can receive awards for the best poster, as decided by peers, a faculty panel, and the community partners.

Technology can be used to highlight student learning, community impact, and the campus-community partnership. Students can use several free websites (e.g., Slideboom, Authorstream, YouTube) to narrate slideshows or videos to be published online. These presentations may be researchfocused, descriptive, or reflective of how the experience has shaped the students' personal and professional identities. Lastly, the Community-Campus Partnerships for Health has organized CES4Health.info, an online mechanism for peerreviewing, publishing, and disseminating products of health-related community-engaged scholarship. Medical students may submit their presentations for possible publication. The possibility of publication may especially attract higher-ranking students to service-learning activities, since they have been found to choose engagement with research, rather than engagement with service-learning. ${ }^{37}$

\section{Discussion}

The changing nature of medicine and its practice begs new approaches to educating future physicians. From this study's review, service-learning, when infused systematically, appears to provide simultaneous opportunities to train students authentically, engage the community and university reciprocally, and develop students' cognitive-emotional dimensions.

The literature on service-learning in medical education revealed that programs across medical schools are not homogeneous. The varieties of service-learning programs reflect the multitude of community needs while also addressing the learning objectives of students at different stages of their training.

A shared theme across these diverse programs, however, is that service-learning typifies authentic learning. That is, students perform real-world tasks that demonstrate meaningful application of essential knowledge and skills. Authenticity offers mastery experiences for future physicians to develop a professional identity by providing opportunities to grow in confidence, self-reliance, and selfunderstanding; to explore and demonstrate their abilities; and to receive supportive feedback and rewards for their actions. It permits alternatives to traditional assessment, through which educators may gain a richer understanding of their students' strengths and challenges. For students, including authentic assessments like needs assessments and reflection activities ultimately support multiple intelligences and varied learning styles. In short, the infusion of servicelearning to balance the traditional instructor-centered methods may help faculty members avoid isolating the 
"material of education ... [that leads] to a diet of predigested materials". 9,10

A value of engagement from service-learning is that medical students may experience transformative learning vis-à-vis cognitive-emotional development. As individuals critically reflect on their assumptions and beliefs, they can change their frames of reference, which can result in a fundamental change in the basic premises of thoughts, feelings and actions. Educators understand through information processing models that "in meaningful and sustained learning, the intellect and emotion are inseparable," whether learning occurs at a traditional classroom or in a more informal setting. ${ }^{42}$ Research has suggested that pride plays a key role in the development of identity and selfesteem, especially in the context of experience of success, and may dispose students to act positively toward learning and seeking similar experiences in the future. ${ }^{43}$ Continued engagement by students would, therefore, advance the call for more community-centered models in which healthcare professionals adopt a population perspective and advocate for the health of the community, rather than approaching individual patients and their pathologies one symptom at a time.

Medical institutions must become engaged with communities in order to collectively meet the needs and goals of all parties involved. In these "communities of practice," engaged universities embrace communities as equal partners who work with, not for, universities in a mutual exchange to discover new knowledge and promote and apply learning. ${ }^{44}$ This collaborative paradigm redefines universities from curators of knowledge to dialectic partners who must reconsider how they operationalize teaching for the benefit of all-"a successful collaborative process [that] enables a group of people and organizations to combine the complementary knowledge, skills and resources so they can accomplish more together than they can on their own." ${ }^{45}$

In the end, a systematic approach will need to be taken in order to engage faculty, students, and the community in the benefits of service-learning. It appears through the developed framework that service-learning follows an iterative process. In such systematic processes, course content is first designed, and then the course is developed, implemented, evaluated, and revised based on the outcomes. It will be necessary to reform curriculum, prepare faculty through training, garner student participation, highlight community needs and assets through community involvement, and obtain the support at the highest levels of medical education and governmental policy. Infusing service-learning in medical school will depend on the dynamic interplay of these factors. The proposed researchbased framework should feasibly guide those wanting to move toward these goals.

\section{Implications and future directions}

This study promises immediate and long-term implications for medical education. First, for those institutions interested in infusing service-learning into its curricula, faculty training is paramount. In addition to faculty learning how to design, implement, and assess service-learning, it is equally important for faculty to use these opportunities to reflect on how their own practices can change to meet the new professional expectations of their students. Further, faculty, students, and community partners must understand the conceptual differences between service-learning and community service. Institutions can quickly support faculty development by providing financial and material resources. Recognition of those engaged in pedagogies of engagement may symbolically lend institutional support.

More long-term directions will be reimagining goals and how to achieve them, including developing assessments to match and complement traditional approaches. Central to this task will be finding additional community partners/sites, perhaps utilizing a snowball sampling approach. International partnerships via study abroad or disaster assistance also appear to be promising transformative collaborative opportunities.

Lastly, this review offers medical education faculty key avenues to investigate as part of their research agendas. With this in mind, as additional studies are conducted on service-learning in medical education, the literature base on which frameworks and recommendations will be broadened. Therefore, future attention should be placed on revisiting the literature as it is published, within a realistic timeframe (e.g., 3-5 years). Furthermore, as a result of discordant studies in the literature, we recommend that future research conducted on service-learning in medical education provide consistent reporting of descriptive variables (i.e., course type, course objectives, duration, service activity, funding, community partners, participants, types of reflection, and type of assessment).

\section{Limitations}

Future research should take into account the following limitations. First, accuracy in the selection of literature may be limited by databases' and/or journals' poorly assigned keywords on which the search was conducted. Second, given that this study is restricted by cost and timeframe concerns, it was impossible to conduct an exhaustive search of all databases. Rather, a focused search of key databases used predominantly in service-learning and medical education was completed. Third, not all relevant manuscripts were obtainable either through lack of availability or database changes. Nonetheless, article selection was outlined in the method's search criteria and manuscript selection and exclusion procedure. 
With this in mind, a fourth limitation is that researcher judgment was made about which texts to ultimately include. The process for these decisions is transparent and outlined in the methods. As is true of all studies of this type, authors' interpretation of the results is their own construction of knowledge, based on the merging of the objective and the subjective epistemological foundations. This is further complicated when analyses are required across a pool of discordant studies, diverse in their design, methods, quality, participants, and conceptual frameworks. Our conclusions assume the included studies were previously subjected to rigorous review standards and that they are, in fact, methodologically adequate.

Lastly, the literature on service-learning in medical education stems significantly from the United States. What we offer here, therefore, is an attempt to provide "generic" findings and themes for international application. However, we acknowledge that uniqueness is the norm and generalization is impossible in reality.

\section{Conclusion}

The purpose of this study was to identify existing empirical literature on service-learning and medical education in order to develop a general framework that faculty, staff, students, and community members can use when developing service-learning to be infused into medical school curricula. A four-stage framework guides the infusion and implementation across varying medical schools' structures, practices, and locations. Service-learning implemented in medical education has the potential to strengthen campuscommunity relations, improve the lives of the underserved, and develop future medical professionals who are skilled at addressing and preventing health problems interwoven into the fabric of communities in need. ${ }^{46}$

Levine argued that new ideas in higher education would inevitably meet four fates: enclaved, diffused, re-socialized, or terminated. ${ }^{47}$ The determination is made by the individual and collective assessment of the faculty and leadership regarding the compatibility of the new idea with current organizational values and conditions. For this reason, and to advocate for enclaved service-learning within medical education, the proposed four-stage framework is purposefully broad and flexible to help medical schools develop an understanding of, intentional design for, implementation of, and assessment for institutionalizing service-learning that is connected to their mission statement. The components of this framework also serve to help faculty mobilize emergency service-learning projects that manifest outside of the standard curricular parameters and academic timeframes. Engagement in communities and partnerships between institutions and communities are unique, and this four-stage framework should assist medical education programs and community organizations to springboard into service-learning, or continue their momentum toward its institutionalization and refinement for students in all years of their medical career preparation.

\section{Conflict of Interest}

The authors declare that they have no conflict of interest.

\section{References}

1. O'Neil E. The changing health-care system and expectations of physicians. In: Seifer S, Hermans K, Lewis J, editors. Creating community responsive physicians: concepts and models for service-learning in medical education. Washington D.C.: American Association for Higher Education; 2000.

2. Berwick DM, Finkelstein JA. Preparing medical students for the continual improvement of health and health care: Abraham Flexner and the new "public interest." Academic Medicine. 2010;85:S56-S65.

3. Irby DM, Cooke M, O’Brien BC. Calls for reform of medical education by the Carnegie Foundation for the Advancement of Teaching: 1910 and 2010. Academic Medicine. 2010;85:220-227.

4. Gupta RP, de Wit ML, McKeown D. The impact of poverty on the current and future health status of children. Paediatr Child Health. 2007;12:667672.

5. Prober CG, Heath C. Lecture halls without lectures-a proposal for medical education. New England Journal of Medicine. 2012;366:1657-1659. 6. Bligh DA. What's the Use of Lectures? San Francisco: Jossey-Bass; 2000. 7. McLaughlin JE, Roth MT, Glatt DM, Gharkholonarehe N, Davidson CA, Griffin LM, et al. The flipped classroom: a course redesign to foster learning and engagement in a health professions school. Academic Medicine. 2014;89:236-243.

8. Billig SH. Research on K-12 school-based service-learning. Phi Delta Kappa. 2000;81:658-664.

9. Dewey J. Experience and education. New York: Collier Books; 1938.

10. Giles D, Eyler J. The theoretical roots of service-learning in John Dewey: toward a theory of service-learning. Michigan Journal of Community Service-learning. 1994;1:77-85.

11. Charmaz K, Mitchell R. Grounded theory in ethnography. Handbook of ethnography. 2001:160-174.

12. Averill N, Sallee J, Robinson J, Mcfarlin J, Montgomery A, Burkhardt G, et al. A first-year community-based service-learning elective: design, implementation, and reflection. Teaching and Learning in Medicine. 2007;19:47-54.

13. Buckner A, Ndjakani Y, Banks B, Blumenthal D. Using service-learning to teach community health: the Morehouse School of Medicine community health course. Journal of the Association of American Medical Colleges. 2010;85:1645-1651.

14. Burrows M, Chauvin S, Lazarus C, Chehardy P. Required servicelearning for medical students: program description and student response. Teaching and Learning in Medicine. 1999;11:223-231.

15. Elam C, Sauer M, Stratton T, Skelton J, Crocker D, Musick D. Servicelearning in the medical curriculum: developing and evaluating an elective experience. Teaching and Learning in Medicine. 2003;13:194-203.

16. Jefferson A, Cantwell N, Byerly L, Morhardt D. Medical student education program in Alzheimer's disease: the PAIRS program. BMC Medical Education. 2012;12:1-8.

17. Leung K, Liu W, Wang W, Chen C. Factors affecting students' evaluation in a community service-learning program. Advances in Health Sciences Education. 2007;12:475-490.

18. Long J, Lee R, Federico S, Battaglia C, Wong S, Earnest M. Developing leadership and advocacy skills in medical students through service-learning. Journal of Public Health Management and Practice 2011;17:369-372. 19. McConnell E, Clasen C, Stolfi A, Anderson D, Markert R, Jaballas E. Community service and the pediatric exam: an introduction to clinical medicine via a partnership between first year medical students and a community elementary school. Teaching and Learning in Medicine. 2010;22:187-190.

20. McNeal M, Buckner A. Using mini-grants and service-learning projects to prepare students to serve underserved populations. Journal of Health Care for the Poor and Underserved. 2012;23:20-26. 
21. Meili R, Fuller D, Lydiate J. Teaching social accountability by making the links: qualitative evaluation of student experiences in a service-learning project. Medical Teacher. 2011;33:659-666.

22. Packer C, Carnell R, Tomcho P, Scott J. Development of a four-day service-learning rotation for third-year medical students. Teaching and Learning in Medicine. 2010;22:224-228.

23. Sakai D, Fukuda M, Nip I, Kasuya R. School health education at the Queen Emma clinics: a service-learning project at the John A. Burns school of medicine. Hawaii Medical Journal. 2002;61:14-17.

24. Steiner B, Sands R. Responding to a natural disaster with servicelearning. Family Medicine. 2000;32:645-649.

25. Stearns J, Stearns M, Glasser M, Londo R. Illinois RMED: a comprehensive program to improve the supply of rural family physicians. Family Medicine. 2000;32:17-21

26. Switzer C. Service-learning in a medical school: psychosocial and attitudinal outcomes. Dissertation. Pittsburg: University of Pittsburg; 1999. 27. O'Toole T, Kathuria N, Mishra M, Schukart D. Teaching professionalism within a community context: perspectives from a national demonstration project. Academic Medicine. 2005;80:339-343.

28. Waddell R, Davidson R. The role of the community in educating medical students: initial impressions from a new program. Education for Health. 2000;13:69-76

29. Wee L, Xin Y, Koh G. Doctors-to-be at the doorstep-comparing servicelearning programs in an Asian medical school. Medical Teacher. 2011;33:471-478.

30. Ferrari N, Cather G. Community service, learning and the medical student. Education for Health-Abingdon-Carfax Publishing Limited. 2000;15:222-227.

31. Stewart T, Alrutz M. Meaningful relationships: cruxes of universitycommunity partnerships for sustainable and happy engagement. Journal of Community Engagement and Scholarship. 2012;5:44-55.

32. Elam C, Musick D, Sauer M, Skelton J. How we implemented a servicelearning elective. Medical Teacher. 2002;24:249-253.

33. Eyler J, Giles D. Where's the learning in service-learning? San Francisco: Jossey-Bass; 1999.

34. Farr N. Service-learning curriculum evaluation and proposal. Master's thesis. Galveston: The University of Texas Medical Branch; 2010.

35. Veronesi M, Gunderman R. Perspective: the potential of student organizations for developing leadership: one school's experience. Academic Medicine. 2012;87:226-229.

36. Cene C, Peek M, Jacobs E, Horowitz C. Community-based teaching about health disparities: combining education, scholarship, and community service. Journal of General Medicine. 2010;25:130-135.

37. Brush D, Markert R, Lazarus C. The relationship between service learning and medical student academic and professional outcomes. Teaching and Learning in Medicine. 2006;18:9-13.

38. Eyler J, Giles D, Schmeide A. A practitioner's guide to reflection in service-learning. Nashville: Vanderbilt University; 1996.

39. Bringle R, Phillips M, Hudson M. The measure of service learning: research scales to assess student experiences. Washington D.C.: APA; 2004.

40. Stewart T. Meta-reflective service-learning poster fairs: purposive pedagogy for pre-service teachers. Journal of the Scholarship of Teaching and Learning. 2008;8:79-102.

41. Mckinney K. The scholarship of teaching and learning: past lessons, current challenges, and future visions. In: Wehlburg C, Chadwick-Blossey S, editors. To improve the academy. Bolton: Anker Publishing; 1999.

42. McCombs BL. The learner-centered psychological principles: a framework for balancing academic and social and emotional learning. Center on Education in the Inner Cities Review, 2001:10;8-9.

43. Ingleton C. Emotion in learning: a neglected dynamic. Proceedings of the Higher Education Research and Development Society of Australasia: Annual International Conference; 1999.

44. Lave J, Wenger E. Situated Learning: legitimate peripheral participation. Cambridge: Cambridge University Press; 1991

45. Torres J. Benchmarks for campus/community partnerships. Providence: Campus Compact; 2000

46. Hunt J, Bonham C, Jones L. Understanding the goals of service learning and community-based medical education: a systematic review. Academic Medicine. 2011;86:246-251.

47. Levine A. Why innovation fails: the institutionalization and termination of innovation in higher education. Albany: State University of New York Press; 1980 